\title{
Efficacy of BioXtra Spray and Mouthwash in Patients with Radiation-induced Xerostomia: A Randomized Clinical Trial
}

\author{
${ }^{1}$ Mahin Bakhshi, ${ }^{2}$ Soheila Manifar, ${ }^{3}$ Nemat Azizi, ${ }^{4}$ Samira Basir Shabestari, ${ }^{5}$ Abbas Hossein Mardi \\ ${ }^{6}$ Iman Shirinbak, ${ }^{7}$ Aida Mehdipour
}

\begin{abstract}
Aim: Xerostomia is an important complication following radiotherapy for head and neck regions producing serious discomfort for patients. Symptomatic treatments for the disease include strict oral hygiene and use of artificial saliva substitutes. The present study compared the efficacy of BioXtra spray and mouthwash in the relief of radiotherapy-induced xerostomia in patients referred to Imam Khomeini Hospital, Tehran University of Medical Sciences.
\end{abstract}

Materials and methods: In a cross-over randomized clinical trial, BioXtra spray and mouth wash were used by 20 patients for 2 weeks with a 1 week wash-out period. The ease of application and the patients' acceptance of the products were obtained using $100 \mathrm{~mm}$ visual analog scale (VAS) and dichotomous scales. We used SPSS version. 18.0 to analyze our data considering level of significance less than 0.05 .

Results: Both forms of BioXtra showed similar effect in reducing oral dryness and its complications. The mean VAS was 33.2 for spray and 34.5 for mouthwash. Majority of our patients reported improvements in xerostomia after using spray (85\%) and mouthwash (70\%), respectively. The patients' acceptance of both modalities was similar and they showed equal tendency to continue the treatments. No significant differences in symptoms relief as well as judgment of the regimens were found between groups.

\footnotetext{
${ }^{1}$ Associate Professor, ${ }^{2,4,6,7}$ Assistant Professor, ${ }^{3}$ General Practitioner, ${ }^{5}$ Dentist

${ }^{1}$ Department of Oral Medicine, Shahid Beheshti University of Medical Sciences, Tehran, Iran

${ }^{2}$ Department of Oral Medicine, Imam Khomeini Hospital, Tehran University of Medical Sciences Tehran, Iran

${ }^{3}$ Educational Organization, Zanjan, Iran

${ }^{4}$ Department of Oral Medicine, Dental School, Zanjan University of Medical Sciences, Zanjan, Iran

${ }^{5}$ Department of Oral Medicine, Dental School, Qazvin University of Medical Sciences, Qazvin, Iran

${ }^{6}$ Department of Maxillofacial Surgery, Dental School, Zanjan University of Medical Sciences, Zanjan, Iran

${ }^{7}$ Department of Pediatric, Dental and Oral Health Research Centre, Qom University of Medical Sciences, Zanjan, Qom, Iran

Corresponding Author: Aida Mehdipour, Assistant Professor Department of Pediatric, Dental and Oral Health Research Centre, Qom University of Medical Sciences, Qom, Iran, Phone: 00982537700094, e-mail: mehdipoor_aida@yahoo.com
}

Conclusion: BioXtra spray and mouthwash are similarly effective in reducing the symptoms of postradiotherapy xerostomia.

Keywords: Xerostomia, Radiation therapy, BioXtra.

How to cite this article: Bakhshi M, Manifar S, Azizi N, Shabestari SB, Mardi AH, Shirinbak I, Mehdipour A. Efficacy of BioXtra Spray and Mouthwash in Patients with Radiationinduced Xerostomia: A Randomized Clinical Trial. Int J Experiment Dent Sci 2014;3(1):19-23.

Source of support: Nil

Conflict of interest: None declared

\section{INTRODUCTION}

Radiotherapy (RT) is a treatment modality which is regularly used in the treatment of the head and neck cancers (HNC). This method destroys tumor cells and also we can expect undesirable effects on normal tissues. ${ }^{1}$ Salivary glands are often involved in the head and neck cancer radiotherapy field because of their position. Radiotherapy destroys salivary gland parenchyma and as a result we can expect varying degrees of xerostomia. This phenomenon is usually permanent. Irreversible effects happen at total dosage of greater than $5000 \mathrm{cGy}^{2}$

Saliva has an important protective role against oral disease. It is necessary for lubrication, regulation of the oral cavity flora, teeth demineralization and maintaining soft and hard tissue integrity. ${ }^{3,4}$

Xerostomia, or dryness of the mouth, can cause oral discomfort and pain, difficulty in mastication and swallowing, speech problems, and impairment of taste and smell. It may also lead to an increased risk of dental caries or oral infections. As a result, nutrition intake can be affected with subsequent weight loss. So xerostomia is a major health issue in these patients. ${ }^{5}$

Xerostomia can be managed symptomatically. Dental caries and oral infections can be prevented appropriately by strict oral hygiene, fluoride agents and antimicrobials. Systemic sialagogues such as pilocarpine, civemiline, anethole trithione and bethanechol could stimulate salivary secretion from remaining functional salivary acini. These agents have side effects on patients with systemic disease. ${ }^{6}$ However, in the absence of functioning tissue, wetting agents or saliva substitutes can be used. These products are mainly based on carboxymethylcellulose (CMC) so these commercials 
are more viscous than saliva and do not stimulate viscoelastic property of saliva. They also lack the immunological protection of the saliva which is mainly due to lysozyme, lactoferrin, lactoperoxidase, and immunoglobulins of the natural saliva. ${ }^{7}$

BioXtra is a new generation of products to relief Xerostomia which includes hydroxyl-ethyl cellulose, xylitol, glucose oxidase, lactoperoxidase, polyglyceryl methacrylate, lactoferrin, lysozyme, monofluorophosphate, and other component of natural saliva. It is introduced in several forms such as spray, mouth wash, gel, toothpaste. ${ }^{8,9}$

Because of lacking study to compare the efficacy of two forms of BioXtra (spray and mouthwash) on radiotherapyinduced Xerostomia, we tried to achieve this goal.

\section{MATERIALS AND METHODS}

Our study was a randomized double blind clinical trial (approved by Ethics Committee of the Research Division of Qazvin University of Medical sciences) and registered on clinical trial. gov: Identifier number: NCT01195233) 20 patients (10 male, 10 female) who were previously subjected to radiotherapy for head and neck cancer at Cancer Institute, Imam Khomeini Hospital, were asked to participate in this study. Coordinator explained the process to each patients and informed consent were signed by them. All the patients agreed to participate. This project was granted approval by Ethics Committee.

Patients with subjective complaint of dry mouth since radiotherapy ( $\geq 50 \mathrm{~Gy}$ ) for $\mathrm{HNC}$ encompassing bilateral major salivary glands, atleast 6 months earlier were eligible to participate. The age of our patients were 18 years or older. They take nutritious orally (i.e. not parenterally or through PEG tube). Patients with a history of cancer recurrence, end stage malignancies, systemic disease that causes xerostomia such as diabetes mellitus, sjogren syndrome were excluded. Patients, who were taking any medications with anticholinergic side effects such as antidepressants or diuretics, were also excluded.

Using random allocation software, all eligible patients allocated in two groups:

The first group was given BioXtra spray for 2 weeks followed by 1 week wash-out period then, they used BioXtra mouthwash for the next 2 weeks. The order of the interventions was changed for the second group (the first 2 weeks, BioXtra mouthwash and after the washout, BioXtra spray was applied).

During the study, application of other products for the treatment of xerostomia such as saliva substitutes or stimulants was not allowed; although, patients could take sips of water frequently. However, they were permitted to use other mouth care systems such as antifungal treatment or topical analgesic agents.

The clinician who visited the patients during the study was different from the one who gave the intervention to the patients. Data collectors and the one who analyzed the data know the groups only as A or B and they did not know the order of drug usage.

Involved patients were asked to fill out two forms of questionnaire at first visit or day 0 . They were also asked to complete two other forms at the second visit (day 14) and the third visit (day 35). Questionnaires which were used was similar to shadad et $\mathrm{al}^{8}$ designed in two forms including VAS and dichotomous scale (yes or no) as below:

Questionnaire for the first day of treatment

Variables recorded based on $100 \mathrm{~mm}$ VAS

1. To what extent your mouth is dry? (Extremes: 0 'not dry at all', $100=$ 'worst imaginable dryness').

2. Do you have difficulty in swallowing because of your dry mouth? (Extremes: $0=$ 'no difficulties at all', $100=$ 'very severe difficulties swallowing').

3. Do you have speech difficulty because of your dry mouth? (Extremes: 0 = 'no difficulties at all', 100 = 'very severe difficulties with speech').

4. Do you have difficulty in chewing as a result of your dry mouth? (Extremes: 0 = 'no difficulties at all', 100 = 'very severe difficulties in chewing').

5. Do you have a burning sensation in your mouth? (Extremes: $0=$ 'no burning at all', 100 = 'very severe burning mouth').

6. Is taste affected by your dry mouth? (Extremes: $0=$ 'not affected at all', 100 = 'very severe alteration of taste').

Questionnaire for the first day of treatment variables recorded based on dichotomous scales (Yes or No)

1. Do you experience a dry mouth in the daytime? Yes No

2. Do you experience a dry mouth during night? Yes No

3. Is the dry mouth worsens in the day rather than Yes No in the night?

4. Do you wake in the night as a result of dry mouth? Yes No

5. If you wear denture, does your dry mouth affect Yes No the retention of it?

6. Do you get a bad taste in your mouth? Yes No

7. Do you avoid speaking to people as a result of Yes No your dry mouth?

8. Do you currently use any kind of saliva substitute? Yes No

9. If yes - do you find it effective? Yes No

Questionnaire for the second and the third visits (day 14 and 35) of treatment

Variables recorded based on $100 \mathrm{~mm}$ VAS

1. How effective was the product system at relieving your dry mouth? (Extremes: 0 = 'not effective at all', $100=$ extremely effective')

2. How pleasant was the taste of the product? (Extremes: $0=$ 'not pleasant at all', 100 = 'extremely pleasant')

3. How pleasant did your mouth feel after using the product system? (Extremes: 0 = 'not pleasant at all', 100 = 'extremely pleasant'). 
Questionnaire for the second and the third visits (Day 14 and 35) of treatment period variables recorded based on Dichotomous scales (Yes or No)

1. Did the product make your dry mouth better? Yes No

2. Did the product make you chew easier? Yes No

3. Did the product make you swallow easier? Yes No

4. Did the product make you talk easier? Yes No

5. Did the product improve your sensation of Yes No taste?

6. Did the product improve the burning sensation? Yes No

7. Would you like to continue using this product? Yes No

8. Did you feel better after using this product? Yes No

9. Was the product easy to use? Yes No

We used SPSS vs 18.0 to analyze our data considering level of significance less than 0.05. Statistical analysis between two forms (spray and mouthwash) was done using Student t-test (for VAS scale) and exact fisher test (for dichotomous scale).

\section{RESULTS}

Our study was performed on 20 patients (10 males and 10 females). Table 1 shows VAS scores recorded for the first question. Mean VAS for each question at first day is reported.

Table 1: Mean VAS scores at the first visit

\begin{tabular}{cllcc}
\hline Question & Mean & SD & Minimum & Maximum \\
\hline 1 & 71.2 & 23.9 & 20 & 100 \\
2 & 56.7 & 35.9 & 0 & 100 \\
3 & 62.0 & 25.2 & 0 & 100 \\
4 & 64.5 & 31.5 & 0 & 100 \\
5 & 41.2 & 34.2 & 0 & 100 \\
6 & 61.0 & 36.1 & 0 & 100 \\
\hline
\end{tabular}

At the beginning of the study, all the patients (100\%) were suffering from xerostomia. A total of 19 patients had dryness at night. Getting bad taste in mouth, effect on denture, avoiding speaking was observed in $16(80 \%), 4(20 \%)$ and $18(90 \%)$ of the patients, respectively. Five patients (25\%) used saliva substitute among them 4 patients $(80 \%)$ found it useful.

By using both forms of BioXtra (spray and mouthwash), we observed improvement in xerostomia and its complications. Results of application of two systems after 14 days listed in Tables 2 and 3.

\section{DISCUSSION}

We showed that BioXtra products (spray and mouthwash), have significant effects on xerostomia related complications along with better acceptance among patients. Both products have the same effects on alleviating xerostomia complications. Their acceptance among patients is similar. These products have the most effect on improvement of oral dryness and speech with least on patients taste.

Xerostomia is considered as a major side-effect following radiotherapy in the field of head and neck. ${ }^{1}$ It can adversely affect patient's quality of life. Oral functions such as ability to sallow, speaking can be violated. Susceptibility to dental caries and oral infections is common among these patients. As a result of impaired lubrication, low tolerance for dental prostheses can be observed. These symptoms can change food choices with subsequent malnutrition. ${ }^{10,11}$

To reduce the severity of oral complications due to xerostomia, aggressive oral care before radiotherapy is recommended. Evaluation of oral health status by a dental team

Table 2: Improvement in xerostomia regarding application of BioXtra spray and mouthwash after 14 days (VAS scales)

\begin{tabular}{|c|c|c|c|c|c|c|}
\hline \multirow[t]{2}{*}{ Outcome } & \multicolumn{2}{|c|}{ Spray } & \multicolumn{2}{|c|}{ Mouthwash } & \multirow[t]{2}{*}{ Mean difference } & \multirow[t]{2}{*}{$p$-value } \\
\hline & Mean & $S D$ & Mean & $S D$ & & \\
\hline Improvement in oral dryness & 33.1 & 21.3 & 35.7 & 29.4 & 2.5 & NS \\
\hline Pleasant taste of product & 41.5 & 30.5 & 43.0 & 30.8 & 1.4 & NS \\
\hline Pleasant feel after usage of product & 39.4 & 28.9 & 39.2 & 31.7 & 0.2 & NS \\
\hline
\end{tabular}

*Independent t-test, NS: No significant

Table 3: Improvement in xerostomia regarding application of BioXtra spray and mouthwash after 14 days (dichotomous scales)

\begin{tabular}{|c|c|c|c|c|c|}
\hline \multirow[t]{2}{*}{ Outcomes } & \multicolumn{2}{|c|}{ Spray } & \multicolumn{2}{|c|}{ Mouthwash } & \multirow[t]{2}{*}{$p$-value* } \\
\hline & Yes & No & Yes & No & \\
\hline Improvement in oral dryness & 18 & 2 & 14 & 6 & NS \\
\hline Improvement in chewing & 13 & 7 & 12 & 8 & NS \\
\hline Improvement in swallowing & 14 & 6 & 12 & 8 & NS \\
\hline Improvement in speech & 17 & 3 & 15 & 5 & NS \\
\hline Improvement in taste & 10 & 10 & 11 & 9 & NS \\
\hline Improvement in burning sensation & 14 & 6 & 12 & 8 & NS \\
\hline Tendency toward continuing treatment & 17 & 3 & 17 & 3 & NS \\
\hline Feel better after usage & 19 & 1 & 15 & 5 & NS \\
\hline Ease of application & 19 & 1 & 17 & 3 & NS \\
\hline
\end{tabular}

${ }^{*}$ Fisher exact test, NS: No significant 
before starting radiotherapy is vital part. Special attention must be given to mucosal lesions, dental caries, periodontal problem and improper dentures. ${ }^{1}$

Current management of xerostomia largely relies on the symptomatic treatment. Pharmacologic management include the use of fluoride agents for optimal oral hygiene, antimicrobials to prevent oral infection, saliva substitutes to overcome dryness, and sialogogic agents to stimulate the remaining salivary gland tissues for saliva production. ${ }^{12,13}$

Saliva substitutes containing hydroxyethyl, hydroxypropyl, or CMC may be helpful by temporarily wetting the oral mucosa to overcome complications of xerostomia. ${ }^{14}$

Jellema et al on a study regarding use of a xanthan gumbased saliva substitutes, showed similar improvement with this salivary substitute and placebo (of similar composition, without xanthan gum). They concluded that this agent was no longer of benefit rather than other saliva substitutes. ${ }^{15}$

BioXtra is a new generation of products to relief xerostomia which includes hydroxyl-ethyl cellulose, xylitol, glucose oxidase, lactoperoxidase, polyglyceryl methacrylate, lactoferrin, lysozyme, monofluorophosphate, and other component of natural saliva. ${ }^{9}$

Shahdad et $\mathrm{al}^{8}$ in a double-blind, cross $=$ over study compared the usefulness of the BioXtra system and bio-thene oral balance system for radiation induced xerostomia in twenty patients. The results showed that BioXtra system is significantly effective in reducing symptoms associated with radiotherapy induced xerostomia. Improvement of the symptoms such as oral dryness, problem in chewing, difficulty in speaking, and swallowing along with changes in taste are significant. Their results showed that the acceptance of the taste of BioXtra products (Tooth paste, mouthwash and gel) were better among patients. ${ }^{8}$

Dirix et $\mathrm{al}^{9}$ in their study evaluated the effect of BioXtra system on 34 patients with radiotherapy induced xerostomia. At the beginning of their study, 19 patients suffer from severe xerostomia which was fallen to nine patients after treatment. Their results showed significant improvement with xerostomia related complications. Although, it was observed to have fewer effects on alleviating oral pain but its effect was significant. The mean VAS score was 59.8 at the beginning of treatment course which reduced to 36.4 at day 28 which was statistically significant $(\mathrm{p}<0.001) .{ }^{9}$

Gookizadeh et al ${ }^{16}$ in their study tried to design a clinical research on BioXtra efficacy for treating xerostomia in 58 patients with head and neck cancer (except salivary gland cancers). In comparison with results before treatment with BioXtra, xerostomia during the day and night and speech impairment was significantly lower after treatment, but xerostomia while eating and drinking water during a meal indicated no significant difference, because in this situation, we need more saliva flow, and BioXtra cannot improve xerostomia with different scores. ${ }^{16}$ Our results are in concordance with previous studies.

Studies on BioXtra system among patients with other cause of xerostomia and also evaluating the effect of BioXtra on bacterial colonization are recommended.

\section{ACKNOWLEDGMENTS}

We would like to thank cancer institute healthcare personals for helping in process of study and also Dr Navid Mohammadi for his sincere effort in statistical analysis of data.

\section{REFERENCES}

1. Warde P, Kroll B, O'Sullivan B, Tew-George E, Aslanidis J, Waldron J, Maxymiw W, Liu FF, Payne D, Cummings B. A phase II study of Biotène in the treatment of postradiation xerostomia in patients with head and neck cancer. Support Care Cancer 2000;8(3):203-208.

2. Epstein J, Van Der Waal I. Oral Cancer. In: Greenberg M, Glick MA Ship J. L Burket's oral medicine: diagnosis and treatment. 11th ed. Hamilton, Ontario: Bc Decker Inc; 2008; p. 153-189.

3. Sreebny LM. Saliva in health and disease: an appraisal and update. Int Dent J 2000;50(3):140-161.

4. Tenovuo J. Antimicrobial agents in saliva-protection for the whole body. J Dent Res 2002;81(12):807-809.

5. Rieger J, Seikaly H, Jha N, Harris J, Williams D, Liu R, McGaw T, Wolfaardt J. Submandibular gland transfer for prevention of xerostomia after radiation therapy. Arch Otolaryngol Head Neck Surg 2005;131(2):140-145.

6. Chambers MS, Garden AS, Kies MS, Martin JW. Radiation Induced Xerostomia in patients with head and neck cancer: pathogenesis, impact on quality of life, and management. Head Neck 2004;26(9):796-807.

7. Epstein JB, Stevenson-Moore P. A clinical comparative trial of saliva substitutes in radiation-induced salivary gland hypofunction. Spec Care Dentist 1992;12(1):21-23.

8. Shahdad SA, Taylor C, Barclay SC, Steen IN, Preshaw PM. A double-blind, crossover study of Biotène Oral balance and BioXtra systems as salivary substitutes in patients with postradiotherapy xerostomia. Eur J Cancer Care 2005;14(4):319-326.

9. Dirix P, Nuyts S, Vander Pourten V, Delaere P, Van den Bogaert W. Efficacy of the BioXtra dry mouth care system in the treatment of radiotherapy-induced xerostomia. Support Care Cancer 2007;15(12):1429-1436

10. Hong CHL, Napeñas JJ, Hodgson BD, Stokman MA, MathersStauffer V, Elting LS, Spijkervet FK, Brennan MT. A systematic review of dental disease in patients undergoing cancer therapy. Support Care Cancer 2010;18(8):1007-1021

11. Logemann JA, Smith CH, Pauloski BR, Rademaker AW, Lazarus CL, Colangelo LA, Mittal B, MacCracken E, Gaziano J, Stachowiak L, et al. Effects of xerostomia on perception and performance of swallow function. Head Neck 2001;23(4): 317-321.

12. Fox PC. Salivary enhancement therapies. Caries Res 2004; 38(3):241-246.

13. Epstein JB, Robertson M, Emerton S, Phillips N, StevensonMoore P. Quality of life and oral function in patients treated with radiation therapy for head and neck cancer. Head Neck 2001; 23(5):389-398. 
14. Leveque FG, Montgomery M, Potter D, Zimmer MB, Rieke JW, Steiger BW, Gallagher SC, Muscoplat CC. A multicenter, randomized, double-blind, placebo-controlled, dose titration study of oral pilocarpine for treatment of radiation-induced xerostomia in head and neck cancer patients. J Clin Oncol 1993; 11(6):1124-1131.

15. Jellema AP, Langendijk H, Bergenhenegouwen L, van der Reijden W, Leemans R, Smeele L, Slotman BJ. The efficacy of
Xialine in patients with xerostomia resulting from radiotherapy for head and neck cancer: a pilot-study. Radiother Oncol 2001; 59(2):157-160

16. Gookizadeh AB, Emami HA, Najafizadeh NA, Roayaei MA. Clinical evaluation of BIOXTRA in relieving signs and symptoms of dry mouth after head and neck radiotherapy of cancer patients at Seyed-al-Shohada Hospital, Isfahan, Iran. Adv Biomed Res 2012;1:72. 